\title{
A IMPORTÂNCIA DO GOLEIRO E SUA PARTICIPAÇÃO TÁTICA COM A BOLA NOS PÉS
}

Wellington Renato Rossi ${ }^{1}$; José Lucas Polizel Fagundes ${ }^{1}$; Lucas da Silva Santos ${ }^{1}$; Guilherme Akio Tamura Ozaki $^{23}$; Everton Alex Carvalho Zanuto ${ }^{1}$; Robson Chacon Castoldi ${ }^{12}$; José Carlos Silva Camargo Filho ${ }^{4}$

${ }^{1}$ Universidade do Oeste Paulista - UNOESTE, Curso de Educação Física, Presidente Prudente, SP. ${ }^{2}$ Universidade Estadual de Campinas - UNICAMP, Faculdade de Ciências Médicas, Campinas, SP. ${ }^{3}$ Universidade Paulista - UNIP, Departamento de Fisioterapia, Campus de Araçatuba, SP. ${ }^{4}$ Universidade Estadual Paulista - FCT/UNESP, Departamento de Fisioterapia, Presidente Prudente, SP.

\section{RESUMO}

O futebol moderno se tornou mais veloz e dinâmico em razão de alterações nas regras. Desse modo, com todas as modificações táticas, o goleiro passou a ter importância ainda maior dentro de sua equipe. Este estudo teve o objetivo de analisar a importância do goleiro em partidas de futebol profissional. Foram analisadas 13 partidas de futebol, divididas em dois grupos. 0 grupo 1 foi composto pela análise de nove partidas disputadas durante a Copa do Mundo FIFA 2014. O grupo 2 foi formado por quatro jogos de outras competições, selecionadas a partir da participação de goleiros com maior habilidade nos pés, conforme ranking de gols da Federação Internacional de História e Estatística do Futebol (IFFHS). Foi realizado o download dos jogos no site de domínio público do Youtube e em seguida, estes foram analisados por meio do software SKOUT, quanto ao posicionamento tático e quantificação dos números de cada goleiro (parte técnica) durante as partidas. Foi utilizado o teste de normalidade de Shapiro-Wilk para a análise de normalidade e em seguida, o teste $t$ de Student para amostras independentes $(p<0,05)$. Foi verificado que os goleiros do Grupo 2 apresentaram maior participação nos fundamentos de passe, recepção, finalização, reposição com a mão, condução e gols marcados $(p<0,05)$. Porém, na média de erros nos fundamentos de passe, recepção e condução as diferenças não foram significativas. Conclui-se que os goleiros do Grupo 2 apresentaram maior participação nos fundamentos que exigiam habilidade com a posse de bola.

Palavras-chave: futebol, goleiro, fundamentos técnicos e táticos.

\section{THE IMPORTANCE OF GOALKEAPER AND ITS TACTICAL PARTICIPATION WITH BALL ON THE FOOTS}

\begin{abstract}
Modern football has become faster and more dynamic due to changes in rules. In this way, with all the tactical modifications, the goalkeeper started to have even greater importance within his team. This study aimed to analyze the importance of the goalkeeper in professional football matches. Thirteen football matches were analyzed, divided into two groups. Group 1 was comprised of the analysis of nine matches played during the 2014 FIFA World Cup. Group 2 was formed by four matches of other competitions, selected from the participation of goalkeepers with greater skill in the feet, according to the ranking of goal of the International Federation of Football History \& Statistics (IFFHS). The games were downloaded on the public domain site of Youtube and then analyzed through the software SKOUT, regarding the tactical positioning and quantification of the numbers of each goalkeeper (technical part) during the matches. The Shapiro-Wilk normality test was used for normality analysis and then the Student's t-test for independent samples $(p<0.05)$. It was verified that the goalkeepers of Group 2 presented greater participation in the fundamentals of pass, reception, finishing, hand replacement, driving and scored goals $(p<0.05)$. However, in the mean of errors in the pass, reception and driving fundamentals the differences were not significant. It is concluded that the goalkeepers of Group 2 presented greater participation in the fundamentals that required skill with the possession of ball.
\end{abstract}

Keywords: soccer, goalkeeper, technical and tactic fundamentals.

\section{INTRODUÇÃO}

Colloq Vitae 2018 mai-ago; 10(2): 47-53. DOI: 10.5747/cv.2018.v10.n2.v231

ISSN 1984-6436/@ 2018 - Publicado pela Universidade do Oeste Paulista.

Artigo Open Access sob uma licença CC BY-NC-ND (http://creativecommons.org/licenses/by-nc-nd/4.0/). 
O futebol moderno se tornou mais veloz e dinâmico por causa de alterações nas regras. De acordo com Voser, Guimarães e Ribeiro ${ }^{1}$, a partir dessas modificações, houve a contribuição para fluência do jogo e a participação do goleiro, assim como a evolução tática do guarda redes.

Com todas as modificações táticas, o goleiro passou a ter importância ainda maior dentro de sua equipe. Segundo Menezes², o goleiro que era o elemento marginal de sua equipe passou a ser um personagem essencial na equação do jogo, na qual, até então, vivia confinado à pequena área, como quase um estranho recolhido em seu ninho.

Para Macari ${ }^{3}$, os arqueiros fazem parte de uma das posições mais especificas do futebol, no qual nos remete a ouvir aquela antiga fabula do futebol que uma boa equipe começa com um bom goleiro. Com a evolução do futebol, novos requisitos foram exigidos para os jogadores, fazendo com que as equipes evoluíssem tecnicamente, e cada jogador, passasse a ter sua devida importância ${ }^{4}$.

Segundo Leal ${ }^{5}$, a função de goleiro foi criada a partir da lei do jogo estabelecida em 1871, dando a função de poder usar as mãos. 0 goleiro, em qualquer esporte, é fundamental para definição do placar, de tal modo que suas falhas são as mais lembradas pelos torcedores ${ }^{6}$.

Para Moino ${ }^{7}$, a atuação do goleiro se destaca no cenário futebolístico devido a seu alto componente decisivo de uma partida, na qual frequentemente se vê estes atletas diretamente ligados ao sucesso ou fracasso de sua equipe. Para Leal ${ }^{5}$, goleiros são os guardiões da meta, da baliza, e muitas vezes nem são citados no enunciado do sistema tático. Alguns autores vão além e afirmam que um bom goleiro é capaz de levar uma equipe de menor expressão a vitória diante de situações de grande adversidade ${ }^{5,8}$.

Quando se assume a função de goleiro, inúmeras dificuldades devem ser superadas, tais como: diversos e exaustivos treinamentos, a incompreensão do torcedor diante de uma falha, a necessidade de autodisciplina, o controle emocional e a solidão da posição em campo ${ }^{9}$. Desse modo, o goleiro torna-se um atleta que precisa ter formação corporal otimizada, pois tem a possibilidade de utilizar qualquer parte do corpo no contato com a bola ${ }^{3,8}$.

A posição de goleiro em relação ao time é de vital importância, sua participação em determinados lances da partida pode ser a consumação de uma vitória ou até mesmo a $\operatorname{derrota}^{10}$. No entanto, apesar da sua importância na equipe, sua escolha nos times iniciantes e categorias de base, se faz a partir da exclusão entre os demais, quase sempre sendo atribuída ao jogador com menor nível técnico².

Nesse sentido, percebe-se que o goleiro passa a ser um jogador com maiores atribuições, inclusive com a bola nos pés, contribuindo de forma substancial para o sucesso da equipe. Além disso, diante deste acontecimento, surge a possibilidade de adequação ao sistema tático e interação desse jogador no esquema de jogo proposto pelo técnico ${ }^{4}$.

Porém, é possível observar que mesmo após a evolução tática do goleiro, existe a escassez de estudos que abordem esta temática, especialmente a respeito da participação com a bola nos pés. Sendo assim, sabendo da importância desse atleta na equipe de futebol, o presente trabalho teve como objetivo comparar a participação de dois grupos de goleiros em partidas de futebol profissional.

\section{METODOLOGIA}

Fonte de informações

Foram analisadas 13 partidas de futebol, divididas em dois grupos. O grupo 1 foi composto pela análise de nove partidas disputadas durante a Copa do Mundo FIFA $2014 \mathrm{com}$ a quantidade de 13 goleiros observados. 0 grupo 2, foi elaborado por quatro jogos de outras competições, selecionados a partir da participação de goleiros que demonstraram maior facilidade para atuar fora do gol e trabalhar com a bolas nos pés. A definição desses goleiros foi realizada a partir do ranking de gols da International Federation of Football History \& Statistics (IFFHS) ${ }^{11}$.

Grupo 1: Análise dos jogos entre Alemanha $x$ Argentina (Goleiros Manuel Neuer e Sergio Romero), Brasil x Holanda (Júlio César e Jasper Cillessen), Inglaterra $x$ Itália (Joe Hart e Salvatore Sirigu), Alemanha $x$ Argélia (Manuel Neuer e Rais M'Bolhi), Holanda x México (Jasper Cillessen e Guillermo Ochoa), EUA x Alemanha (Tim Howard e Manuel Neuer), Costa Rica x Grécia (Keylor Navas e Orestis Karnezis), Argentina $x$ Bélgica (Sergio Romero e Thibaut Courtois), Colômbia $x$ Uruguai (David Ospina e Fernando Muslera).

Grupo 2: Análise dos jogos entre Alemanha x Colômbia (Goleiro René Higuita - Copa do Mundo FIFA de 1990), São Paulo Futebol Clube $x$ Tigres (Goleiro Rogério Ceni - Libertadores da 
América de 2005), FC Bayern Munich vs Real Madrid (Goleiro Manuel Neuer - Copa Audi de 2015) e Brasil x México (Goleiro Jorge Campos Copa das Confederações de 1998).

Análise dos jogos

Foram efeituados downloads dos jogos de futebol no site de domínio público Youtube (www.youtube.com) para a realização das análises das partidas. A identificação dos fundamentos foi realizada com o software SKOUT no computador que possibilitou leitura, quantificação e análise dos posicionamentos táticos e técnicos.

Critérios para realização das análises

Os critérios dos fundamentos analisados no software SKOUT foram definidos da seguinte forma (Quadro 1 e Figura 1).

Quadro 1. Fundamentos analisados durante os jogos.

\begin{tabular}{lcc}
\hline Fundamentos & Figura & Cor \\
\hline Passe & Círculo & Branco \\
Drible & Círculo & Preto \\
Recepção & Círculo & Amarelo \\
Finalização & Círculo & Azul \\
Lançamento & Círculo & Vermelho \\
Reposição Pé & Triangulo & Preto \\
Reposição Mão & Triangulo & Branco \\
Defesa Fácil & Quadrado & Branco \\
Defesa Difícil & Quadrado & Vermelho \\
Saída de Gol & Quadrado & Azul \\
Desarme & Cruz & Preto \\
Condução & Sinal de Positivo & Amarelo \\
Falta & Losango & Branco \\
Gol & Estrela & Vermelho \\
\hline
\end{tabular}

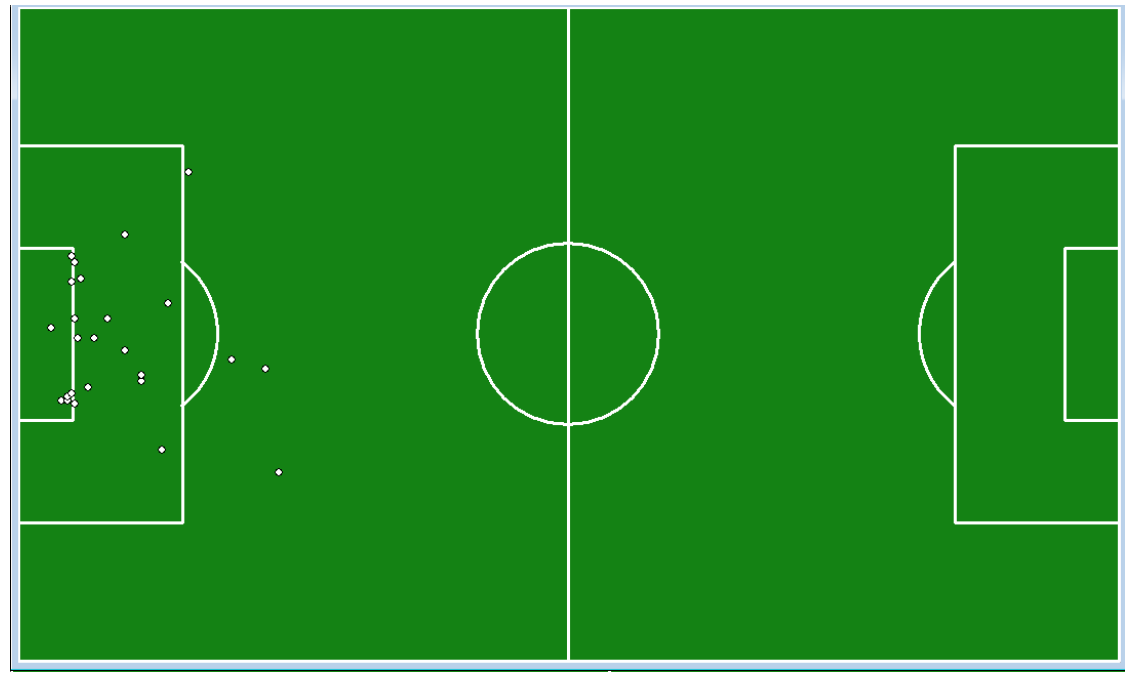

Figura 1. Exemplo de análise do fundamento de passe.

\section{Análise estatística}

Após a obtenção dos resultados, foi utilizada a estatística descritiva para o cálculo dos valores de média e desvio padrão dos dois diferentes grupos estabelecidos. Em seguida, foi utilizado o teste de Shapiro-Wilk para análise de normalidade dos dados e teste $t$ de Student para amostras independentes para comparação entre médias dos dois grupos. Todos os procedimentos foram realizados com o Software SPSS $22.0 \mathrm{com}$ significância $p<0,05$.

\section{RESULTADOS}

Foi verificado que os goleiros do Grupo 2 apresentaram maiores valores médios quando comparado o total de fundamentos (Tabela 1). Nesse Caso, foi observada diferença 
estatisticamente significativa nos fundamentos bola $(p<0,05)$. de passe, recepção, finalização e condução de

Tabela 1. Total de fundamentos analisados a partir de cada grupo.

\begin{tabular}{lcccc}
\hline Fundamentos & $\begin{array}{c}\text { Grupo 1 } \\
\text { (N=13) }\end{array}$ & Média \pm DP & $\begin{array}{c}\text { Grupo 2 } \\
(\mathbf{N}=\mathbf{4})\end{array}$ & Média \pm DP \\
\hline Passe & 112 & $7,47 \pm 4,88$ & 101 & $12,63 \pm 6,02^{*}$ \\
Drible & 1 & $0,07 \pm 0,26$ & 3 & $0,38 \pm 0,74$ \\
Recepção & 126 & $8,40 \pm 3,85$ & 110 & $13,75 \pm 7,19^{*}$ \\
Finalização & 0 & $0,00 \pm 0,00$ & 3 & $0,38 \pm 0,74^{*}$ \\
Lançamento & 154 & $10,27 \pm 7,44$ & 64 & $8,00 \pm 4,54$ \\
Reposição Pé & 25 & $1,67 \pm 2,06$ & 14 & $1,75 \pm 1,98$ \\
Reposição & & $4,20 \pm 2,48$ & 54 & $6,75 \pm 4,10$ \\
Mão & 63 & $3,67 \pm 1,99$ & 46 & $5,75 \pm 4,33$ \\
Defesa Fácil & 55 & $4,13 \pm 3,18$ & 16 & $2,00 \pm 1,69$ \\
Defesa Difícil & 62 & $2,00 \pm 1,60$ & 19 & $2,38 \pm 2,39$ \\
Saída de Gol & 30 & $0,13 \pm 0,35$ & 11 & $1,38 \pm 2,13$ \\
Desarme & 2 & $6,33 \pm 6,66$ & 131 & $16,38 \pm$ \\
Condução & 95 & $0,20 \pm 0,56$ & 2 & $0,25 \pm 0,46$ \\
Falta & 3 & $1,13 \pm 0,92$ & 8 & $1,00 \pm 1,20$ \\
Gol Sofridos & 17 & &
\end{tabular}

Teste $t$ de Student com significância de $5 \% .\left(^{*}\right)$ : p<0,05.

Quando comparados os fundamentos realizados com êxito, percebe-se que os goleiros do grupo 2 demonstraram maiores valores médios para os fundamentos de passe, recepção finalização, reposição com a mão, condução e gols marcados $(p<0,05)$ (Tabela 2$)$.

Tabela 2. Comparação entre os fundamentos realizados com êxito nos diferentes grupos observados.

\begin{tabular}{lcccc}
\hline Fundamentos & $\begin{array}{c}\text { Grupo 1 } \\
\text { (N=13) }\end{array}$ & Média \pm DP & $\begin{array}{c}\text { Grupo 2 } \\
(\mathbf{N = 4 )}\end{array}$ & Média \pm DP \\
\hline Passe & 112 & $7,47 \pm 4,88$ & 100 & $12,50 \pm 6,19^{*}$ \\
Drible & 1 & $0,07 \pm 0,26$ & 2 & $0,25 \pm 0,46$ \\
Recepção & 126 & $8,40 \pm 3,85$ & 110 & $13,75 \pm 7,19^{*}$ \\
Finalização & 0 & $0,00 \pm 0,00$ & 3 & $0,38 \pm 0,74^{*}$ \\
Lançamento & 120 & $8,00 \pm 6,27$ & 49 & $6,13 \pm 3,48$ \\
Reposição Pé & 21 & $1,40 \pm 1,72$ & 11 & $1,38 \pm 1,69$ \\
Reposição Mão & 63 & $4,20 \pm 2,48$ & 54 & $6,75 \pm 4,10^{*}$ \\
Defesa Fácil & 55 & $3,67 \pm 1,99$ & 46 & $5,75 \pm 4,33$ \\
Defesa Difícil & 62 & $4,13 \pm 3,18$ & 16 & $2,00 \pm 1,69$ \\
Saída de Gol & 28 & $1,87 \pm 1,55$ & 20 & $2,50 \pm 2,67$ \\
Desarme & 2 & $0,13 \pm 0,35$ & 11 & $1,38 \pm 2,13$ \\
Condução & 95 & $6,33 \pm 6,66$ & 131 & $16,38 \pm$ \\
Falta & 3 & $0,20 \pm 0,56$ & 2 & $0,25 \pm 0,46$ \\
Gols Marcados & 0 & $0,00 \pm 0,00$ & 3 & $0,38 \pm 0,74^{*}$ \\
\hline
\end{tabular}

Teste $t$ de Student com significância de 5\%. $\left(^{*}\right): p<0,05$. 
O presente estudo também procurou observar os fundamentos sem êxitos nos dois grupos de goleiros. Foi verificado que o Grupo 2 demonstrou maiores valores de erros para as variáveis passe, recepção e condução (Tabela 3). No entanto, não foi verificada diferença estatisticamente significativa $(p>0,05)$.

Tabela 3. Comparação entre os fundamentos realizados sem êxito nos diferentes grupos observados.

\begin{tabular}{lcccc}
\hline Fundamentos & $\begin{array}{c}\text { Grupo 1 } \\
\text { (N=13) }\end{array}$ & Média \pm DP & $\begin{array}{c}\text { Grupo 2 } \\
\text { (N=4) }\end{array}$ & Média \pm DP \\
\hline Passe & 0 & $7,47 \pm 4,88$ & 1 & $12,50 \pm 6,19$ \\
Drible & 0 & $0,07 \pm 0,26$ & 1 & $0,25 \pm 0,46$ \\
Recepção & 0 & $8,40 \pm 3,85$ & 0 & $13,75 \pm 7,19$ \\
Finalização & 0 & $0,00 \pm 0,00$ & 0 & $0,38 \pm 0,74$ \\
Lançamento & 34 & $8,00 \pm 6,27$ & 15 & $6,13 \pm 3,48$ \\
Reposição Pé & 4 & $1,40 \pm 1,72$ & 3 & $1,38 \pm 1,69$ \\
Reposição & 0 & $4,20 \pm 2,48$ & 0 & $6,75 \pm 4,10$ \\
Mão & 0 & $3,67 \pm 1,99$ & 0 & $5,75 \pm 4,33$ \\
Defesa Fácil & 0 & $4,13 \pm 3,18$ & 0 & $2,00 \pm 1,69$ \\
Defesa Difícil & 2 & $1,87 \pm 1,55$ & 0 & $2,50 \pm 2,67$ \\
Saída de Gol & 0 & $0,13 \pm 0,35$ & 0 & $1,38 \pm 2,13$ \\
Desarme & 0 & & & $16,38 \pm$ \\
Condução & 0 & $0,20 \pm 0,56$ & 0 & $0,25 \pm 0,46$ \\
Falta & 18 & $0,00 \pm 0,00$ & 5 & $0,38 \pm 0,74$ \\
Gol Sofridos & & & & \\
\hline
\end{tabular}

Teste t de Student com significância de $5 \%$. Diferenças não significativas $(p>0,05)$.

\section{DISCUSSÃO}

O objetivo do presente estudo foi comparar os fundamentos de goleiros durante partidas de futebol. Nesse caso, foi feita a observação de 13 goleiros da Copa do Mundo FIFA 2014 (Grupo 1) e de quatro goleiros que demonstram maior facilidade para atuar fora do gol e trabalhar com a bolas nos pés, definidos a partir do ranking do IFFHS (Grupo 2).

Foi verificado que os goleiros do Grupo 2 apresentaram maior média quando comparado o total de fundamentos. Nesse Caso, foi observado diferença estatisticamente significativa nos fundamentos de passe, recepção e condução de bola $(p<0,05)$.

Percebe-se que os goleiros do grupo demonstraram maior envolvimento com a posse de bola. Tala fato deve-se provavelmente a maior utilização do goleiro na função de líbero. Além disso, modificações na regra do esporte podem ter colaborado para a maior participação de goleiros com a posse de bola durante as partidas.

Para Barbieri, Benites e Neto ${ }^{9}$, as alterações na regra de impedimento foram as que promoveram mais mudanças nos sistemas de jogo, mesmo que a dinâmica tenha sido pouco alterada. Esta alteração da regra trouxe evolução para os goleiros de modo a permitir uma melhora da tática defensiva, possibilitando os zagueiros fazerem uma linha de marcação mais avançada, uma vez que o goleiro passou a fazer uso da cobertura como líbero, tendo que efetuar maior número de ações fora da área. Com isso, evitando a realização do contra-ataque pelo adversário.

No estudo de Gallo et al. ${ }^{8}$, foi verificado maior percentual de erro nas ações ofensivas realizadas pelos goleiros. Os autores defenderam que se deve direcionar os treinos valorizando o trabalho de reposição de bola pelos goleiros com a finalidade de otimizar as jogadas de ataque.

Quando comparados os fundamentos realizados com êxito, percebe-se que os goleiros do grupo dois demonstraram maiores valores médios para os fundamentos de passe, recepção finalização, reposição com a mão, condução e gols marcados $(p<0,05)$. Nesse cenário, foi possível notar que além de demonstrarem 
maiores valores médios para estas variáveis, dentre todos os fundamentos analisados finalização e gols marcados, estiveram presentes somente no Grupo 2. Provavelmente, a maior habilidade dos goleiros em jogar com a bola nos pés, foi o fator responsável por tal acontecimento.

Apesar da maior obrigação do goleiro ser a realização da defesa, observa-se que esse jogador necessita de capacidades condicionantes e coordenativas, capacidades físicas e técnicas específicas e deve dominar seus fundamentos técnicos ${ }^{13,14}$. Nesse caso, o atributo de jogar com os pés, passa a ser mais uma dentre várias obrigações desse jogador.

Exige-se de um goleiro tal acúmulo de capacidades corporais e psíquicas que só podem ser encontradas em poucos jogadores. A posição do goleiro requer um ensino muito especial e diversos dos demais jogadores ${ }^{11}$.

De acordo com Gonçalves e Nogueira ${ }^{12}$, sabe-se que o treinamento físico é um dos mais importantes ingredientes para o alcance do máximo desempenho esportivo, pois repercute na elevação dos níveis de performance técnicos, táticos, psicológicos e, obviamente, físicos. Já para Maximo ${ }^{13}$, os aspectos psíquicos do goleiro devem ser de tranquilidade, coragem, força de vontade, confiança e de capacidade de reações sequenciais.

Por último, o presente estudo procurou observar os fundamentos sem êxitos nos dois grupos de goleiros. Foi verificado que apesar de não apresentar diferença significativa $(p>0,05), 0$ Grupo 2 demonstrou maiores valores de erros para as variáveis passe, recepção e condução. Tal fato se deve provavelmente ao uso desse recurso, uma vez que apresentam maior habilidade com a posse de bola.

Atualmente existe um impasse entre os especialistas no que diz respeito às habilidades dos goleiros, principalmente no que se refere qual deles saiba jogar melhor com a bola nos pés

\section{REFERÊNCIAS}

1. Voser RC, Guimarães MGV, Ribeiro ER. Futebol: história, técnica e treino de goleiro. Porto Alegre: EDIPUCRS; 2010.

2. Menezes SLC. Rogério Ceni: o goleiro artilheiro: projeto experimental do Curso de Comunicação e fora da área. Dentre estes, destacam-se o brasileiro Rogério Ceni e o alemão Manuel Neuer. $\mathrm{Cabral}^{14}$ discorre que o alemão Manuel Neuer é reconhecido como um goleiro moderno, que utiliza muito bem o jogo com os pés, e que tem um ótimo senso de posicionamento, o tornando em muitas ocasiões da partida um verdadeiro líbero. Já Menezes ${ }^{2}$ descreve Rogério Ceni como atleta diferenciado; completo como goleiro, habilidoso com a bola nos pés, e que seu pioneirismo de goleiro artilheiro vai ficar para a história do futebol brasileiro e mundial.

Nesse sentido, parece haver comum acordo entre estudiosos de que o goleiro que possui habilidade com a posse de bola, pode contribuir para o rendimento da equipe. Desta forma, a inserção do goleiro no sistema de jogo e formação tática, pode ser um recurso de auxílio para o técnico da equipe.

Sendo assim, o presente estudo contribui com a literatura ao analisar de forma específica o posicionamento e ações de goleiros profissionais de alto nível competitivo. No entanto, algumas limitações devem ser consideradas, como a utilização de apenas uma parcela de jogadores participantes da Copa do Mundo FIFA 2014 e goleiros considerados com maiores habilidades com a posse de bola. Estudos que possam investigar campeonatos em diferentes continentes, como a UEFA Champions Leage e a Libertadores da América, podem vir a colaborar com a presente pesquisa.

\section{CONCLUSÃO}

Conclui-se que os goleiros do Grupo 2 apresentaram maior participação nos fundamentos de passe, recepção finalização, reposição com a mão, condução e gols marcados. No entanto, provavelmente por fazer maior uso desses recursos, estes goleiros também apresentaram maior média de erros nos fundamentos de passe, recepção e condução.

Social. Universidade de Juiz de Fora: UFJF; FACOM, 2006.

3. Macari R. Análise cinemática do salto do goleiro de futebol em cobranças de pênaltis: relação entre preferência lateral e desempenho. [Dissertação]. Ribeirão Preto: Faculdade de 
Medicina de Ribeirão Preto, Universidade de São Paulo, 2015. $75 \mathrm{f}$.

4. Tavares O, Telles S. A preparação de goleiros e sua evolução. Rio de Janeiro: Boletim Educação Física; 2006.

5. Leal JC. Futebol arte e oficio. Rio de Janeiro: Sprint, 2000.

6. Freire JB. Pedagogia do esporte. Campinas: Autores Associados, 2003.

7. Moino GS. Análise tática da exigência de situações de jogo para goleiros jovens. Rev Bras Futsal Futebol. 2011;3(8):127-41.

8. Gallo C, Zamai CA, Vendite L, Libardi CA. Análise das ações defensivas e ofensivas, e perfil metabólico da atividade do goleiro de futebol profissional. Conexões: Educ Física, Esporte e Saúde. 2010;8(1):16-37. DOI: https://doi.org/10.20396/conex.v8i1.8637753

9. Barbieri FA, Benites LC, Souza Neto S. Os sistemas de jogo e as regras do futebol: considerações sobre suas modificações. Motriz. 2009;15(2):427-35.

10. Domingues A. Goleiro: 100 segredos. Curitiba: CR\&C Comunicações; 1997.

11. Berto ESM, Magalhães FC. O. Estatura como critério de seleção na captação e formação do goleiro de futebol de campo. Rev Bras Futsal Futebol. 2014;6(20):88-94.

12. Gonçalves G, Nogueira R. O treinamento específico para goleiros de futebol: uma proposta de macrociclo. Rev Estudos Goiânia. 2006;33(7/8):531-43. DOI: http://dx.doi.org/10.18224/est.v33i4.136

13. Máximo AS. Perfil dos preparadores de goleiro de futebol de campo em clubes profissionais da cidade de Florianópolis (categoria de base). Santa Catarina: Universidade Federal de Santa Catarina, 2012.

14. Cabral LFL. Análise das seleções brasileira e alemã de futebol na copa do mundo de 2014. Rio Grande do Sul: Universidade Federal do Rio Grande do Sul, 2015.
Recebido para publicação em 11/07/2017

Revisado em 20/04/2018

Aceito em 18/06/2018 